\title{
Nemaline myopathy: a report of two siblings as evidence of autosomal recessive inheritance of the infantile type
}

\author{
J.D. Cartwright ${ }^{1}$, D.J. Castle ${ }^{2 *}$, M.G. Duffield ${ }^{3}$ and I. Reef ${ }^{1}$ \\ 'Department of Paediatrics, Johannesburg Hospital, and Departments of ${ }^{2}$ Human Genetics and ${ }^{3}$ Pathology, \\ South African Institute for Medical Research, Johannesburg, South Africa
}

\begin{abstract}
Summary: We report two opposite-sex siblings with the severe infantile form of nemaline myopathy; diagnoses were made on muscle biopsy. Neither parent showed clinical or electromyographic evidence of myopathy, and both had negative muscle biopsies. Autosomal recessive inheritance seems likely.
\end{abstract}

\section{Introduction}

Two forms of nemaline myopathy have been described. The adult type is mild, and manifestation is usually delayed until adolescence or later; inheritance is usually autosomal dominant. ${ }^{1}$ The infantile form of the disease presents in the neonatal period, and is usually fatal within the first months of life, with no reported case having survived beyond five years of age. ${ }^{2}$ Although inheritance of this severe form has usually been assumed to be autosomal recessive, there are few reports of affected siblings, and some authors have contested that isolated cases might merely be a severe manifestation of the autosomal dominant variety. ${ }^{3}$

We report two siblings with the infantile form of this disorder. Both parents had muscle biopsies which were negative for nemaline rods. This suggests that an autosomal recessive form of nemaline myopathy does indeed exist. There are implications for genetic counselling in such families.

\section{Case reports}

Case 1

MT-a girl, was the product of an uneventful pregnancy. Birth was by Caesarian section for poor progression of labour, and fetal distress. The infant required active resuscitation, and was placed in an intensive care unit because of respiratory distress; ventilation was not required. Examination revealed a $3.67 \mathrm{~kg}$ infant of normal length and head cir-

Correspondence and *present address: D.J. Castle, M.B., Ch.B., The Maudsley Hospital, Denmark Hill, London, SE5 8AZ, UK.

Accepted: 19 February 1990 cumference. She had a high-arched palate with a 'goldfish' type mouth and expressionless facies, but no other dysmorphic features. There was generalized muscle weakness and hypotonicity, with marked head lag; deep tendon reflexes were depressed. No muscular fasciculations were noted. Cranial nerves were normal, although swallowing was weak, and gag reflex diminished. The rest of the examination was normal.

She smiled at 7 weeks of age, but motor milestones were markedly delayed. At the age of 4 months she contracted a right lower lobe pneumonia, and died after 6 days despite physiotherapy, oxygen, and antibiotics.

Investigations included normal full blood count, serum chemistry, immunoglobulins, and thyroid studies. Aldolase, creatine kinase, and ammonia and amino acids in blood and urine were likewise normal. Barium swallow showed severe gastrooesophageal reflux, and chest $\mathrm{X}$-ray revealed right lower lobe consolidation.

At post-mortem, bilateral bronchopneumonia was present. The brain showed mild hypoxic changes. The rest of the examination was normal apart from the muscle changes. Gomori trichrome stain of skeletal muscle showed red rod-like bodies within the cytoplasm (Figure 1); electron microscopy confirmed them to be nemaline rods, with an electron-dense central zone surrounded by filaments.

\section{Case 2}

JT-during this pregnancy, the mother had noted poor fetal movements, but prenatal ultrasound had revealed no abnormality. Birth was by elective Caesarian section. Apgar scores were $1 / 10$ and $8 / 10$ at 1 and 5 minutes respectively. Early neonatal course was complicated by episodic cyanosis after feeding. 


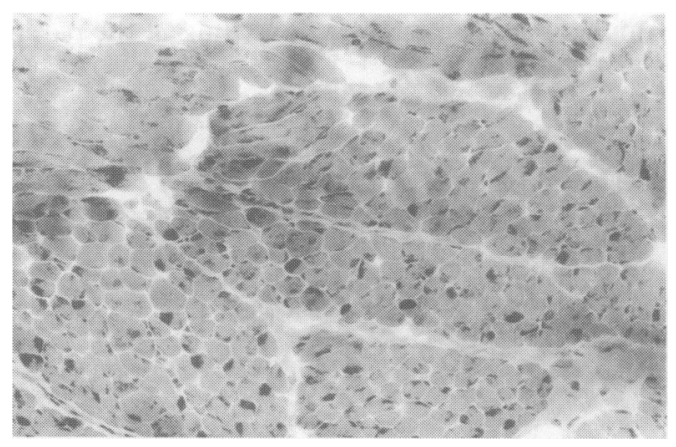

Figure 1 Case 1. Light microscopy: Gomori trichrome stain $\times 18$. Shows nemaline material in autopsy specimen of muscle.

Examination at 3 days of age revealed a vigorous infant of $3.68 \mathrm{~kg}$. He too had a high-arched palate, expressionless facies, and 'goldfish' type mouth. Moro's reflex and grasp were normal, but head lag was marked. There was generalized hypotonicity, and a poor swallowing reflex, but the rest of the examination was normal.

Investigations included normal blood count and serum chemistry. Thyroid function was normal. A muscle biopsy of the right vastus lateralis muscle showed numerous nemaline rods (Figure 2).

The child was discharged at 4 weeks of age to the care of his parents. Initially he required nasogastric feeding, regular chest physiotherapy, and suction; he continued to require tube feeding and suctioning until the age of one year. He has had a number of episodes of pneumonia, which have responded to antibiotic therapy. When last seen, at the age of 2 years 3 months, he was moving his limbs vigorously, sitting adequately by himself, and managing to walk in a walking ring.

\section{Family history}

The parents are not knowingly consanguineous. The father had been adopted at an early age, and does not know his biological parents; the mother has no family history of any muscular diseases, or of early childhood deaths. The parents themselves showed no evidence of muscle weakness, and electromyography (EMG) was normal. Muscle biopsies on both were examined with Gomori's trichome staining and under electron microscopy, and were negative for nemaline rods.

\section{Discussion}

Nemaline myopathy is a recognized pathological entity characterized by the presence of rod-like bodies on electron microscopy of involved muscle. ${ }^{1}$

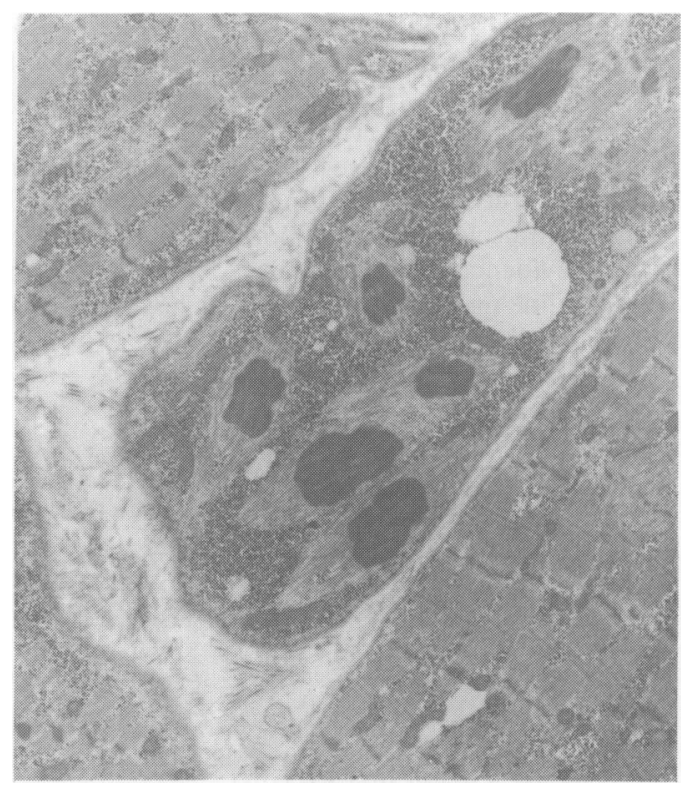

Figure 2 Electron micrograph showing the presence of subsarcolemmal nemaline bodies. Case 2-magnification $\times 2420 ; 20 \mathrm{~mm}$ bar $=3.6 \mu \mathrm{m}$.

Autosomal dominant inheritance has been documented in a number of families. ${ }^{3}$ That an autosomal recessive form of the disease might also exist has been suggested by some authors, ${ }^{4-6}$ and supported by the finding of at least 4 affected individuals with consanguineous parents. ${ }^{7}$ However, Kondo and Yuasa, ${ }^{3}$ in a review of 44 pedigrees, concluded that all could be explained on the basis of autosomal dominant inheritance with variable expression. The present family supports the suggestion of an autosomal recessive form of the disease in that there is no family history, and neither parent had clinical or EMG evidence of myopathy, and both had negative muscle biopsies.

As far as we are aware, there are only three reports of more than one sibling in one generation with nemaline myopathy. ${ }^{8}$ All these children were severely affected. Dysmorphic features similar to those in our patients were consistent features in these children, and generalized muscle weakness in the neonatal period, with conspicuous involvement of the bulbar musculature, was striking. Respiratory assistance and tube feeding or gastrostomy have been necessary in most. Involvement of the intercostal muscles and of the diaphragm, predisposes to frequent bouts of pneumonia in these infants, and death usually occurs in the first year of life. .,8-10 $^{4}$

Reports of muscle biopsies of parents of children with infantile nemaline myopathy are scarce. ${ }^{5}$ In some cases such biopsies have revealed occasional 
nemaline rods, and this has been suggested to be evidence of an autosomal dominant condition with variable expression; however, one might as readily conclude that they indicate carrier status.

In summary, we suggest that the present report of two severely affected siblings, in association with negative muscle biopsies in both parents, provides strong evidence that an autosomal recessive form of nemaline myopathy occurs. This has significant

\section{References}

1. Shy, G.M., Engel, W.K., Somers, J.E. \& Wanko, T. Nemaline myopathy: a new congenital myopathy. Brain 1963, 86: $78-110$.

2. Shafiq, S.A., Dubowitz, V., Petersen, H. de C. \& Milhorat, A.T. Nemaline myopathy: report of a fatal case with histochemical and electron microscopic studies. Brain 1967, 90: 817-828.

3. Kondo, K. \& Yuasa, T. Genetics of congenital nemaline myopathy. Muscle Nerve 1980, 3: 308-315.

4. McComb, R.D., Markesberry, W.R. \& O'Connor, W.N. Fatal neonatal nemaline myopathy with multiple congenital anomalies. $J$ Pediatr 1979, 94: 47-51.

5. Arts, W.F., Bethler, J., Dingemans, K.P. \& Eriksson, A.W Investigations on the inheritance of nemaline myopathy. Arch Neurol 1978, 35: 72-77. implications for genetic counselling of the parents of such children.

\section{Acknowledgements}

We thank Professor Pierre Bill of the University of Natal for his helpful advice. DC acknowledges the support of a Medical Research Council Scholarship.

6. Karparti, G., Carpenter, S. \& Andomann, F. A new concept of childhood nemaline myopathy. Arch Neurol 1971, 24: 291-304.

7. Arts, W.F. \& De Groot, C.J. Congenital nemaline myopathy: two patients with consanguineous parents, one with a progressive course. J Neurol 1983, 230: 123-130.

8. Neustein, H.B. Nemaline myopathy: a family study with three autopsied cases. Arch Pathol 1973, 96: 192-195.

9. Tsuijihata, M., Shimomura, C., Yoshimura, T. et al. Fatal neonatal nemaline myopathy: a case report. $J$ Neurol Neurosurg Psychiatry 1983, 46: 856-859.

10. Kolin, I.S. Nemaline myopathy: report of a fatal case. Am J Dis Child 1967, 114: 95-100. 